EPJ Web of Conferences 52, 06005 (2013)

DOI: $10.1051 /$ epjconf/20135206005

(C) Owned by the authors, published by EDP Sciences, 2013

\title{
Status of JEM-EUSO and its test experiments EUSO-Balloon and TA-EUSO
}

\author{
Andreas Haungs ${ }^{1, \text { a }}$ for the JEM-EUSO Collaboration ${ }^{2}$ \\ ${ }^{1}$ Karlsruhe Institute of Technology - KIT, Institut für Kernphysik, Germany \\ ${ }^{2}$ see http://jemeuso.riken.jp
}

\begin{abstract}
The JEM-EUSO mission will explore the origin of the extreme energy cosmic rays (EECRs) through the observation of their arrival directions and energies. The super-wide-field telescope looks down from the International Space Station onto the night sky to detect UV photons (fluorescence and Cherenkov photons) emitted from air showers generated by EECRs in the Atmosphere. Such a space detector offers the opportunity to observe a huge volume of atmosphere at once and will achieve unprecedented statistical accuracy within a few years of operation. The JEM-EUSO mission will be installed on the Japanese module of the International Space Station. Two test experiments are currently prepared; one to observe the fluorescence background from the edge of the Atmosphere (EUSO-Balloon), and the other to demonstrate, on ground, the capability of all sub-systems of the EUSO instrument (TA-EUSO). In this paper a short review on the scientific objectives and an update of the instrument definition, performances and status of the mission, as well as of the status of the two preceded test experiments will be given.
\end{abstract}

\section{JEM-EUSO and its science}

The least explored region of the wide spanning cosmic ray energy spectrum is the one of the highest energies; i.e. the Extreme Energy Cosmic Rays - EECR. This is natural as the flux of such particles reaching Earth is extremely low. However, in addition to the low statistical accuracy, the existing measurements of various experiments disagree in their flux by a surprisingly large amount (see Fig. 1).

The origin of the differences is still not fully understood and several factors may play a role caused by astrophysics, or reconstruction and detection methods. One of these factors lies probably in the fact that the energy reconstruction is limited by our current theoretical understanding of extensive air showers (EAS). These showers are generated by interaction of the impinging cosmic particle with the Atmosphere. The products of this and subsequently hadronic and electromagnetic interactions are measured with detectors of large exposure and the data is interpreted in terms of the arrival direction, energy and mass of the primary particle. The relevant energies as well as partly the kinematic features of these interactions are not well measured by accelerators, so that extrapolations are used in simulating the EAS leading to different interpretations if different hadronic interaction models or different observables are used.

For the future, large efforts have to be made to reach a higher statistics in measurements, what requires a much higher exposure than that of existing experiments.

The Extreme Universe Space Observatory (EUSO) (Fig. 2) at the Japanese Module (JEM) of the International

\footnotetext{
ae-mail: haungs@kit.edu
}

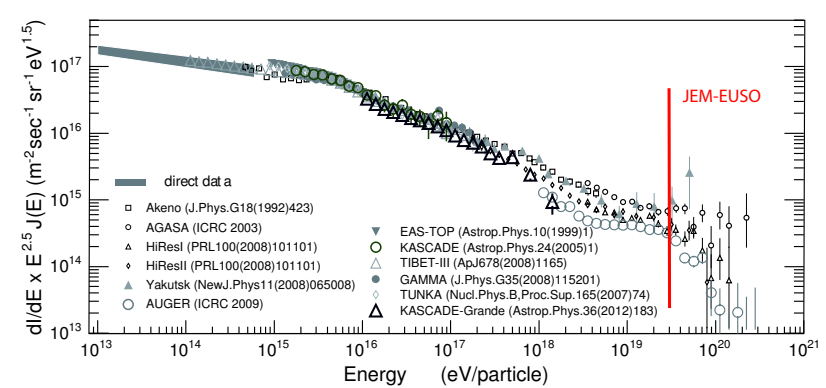

Figure 1. The all-particle spectrum of cosmic rays as measured by various experiments. Largest uncertainties are in the region of the highest energies: the target of the JEM-EUSO mission.

Space Station (ISS) is the first space mission devoted to the scientific research of cosmic rays of highest energies [14]. Main goal is the exploration of the Universe through the detection of the extreme energy cosmic rays and neutrinos by looking downward from the ISS to detect the fluorescence light of extensive air-showers that they generate in the Earth's atmosphere.

The JEM-EUSO instrument is designed to achieve as a main scientific objective astronomy and astrophysics through the particle channel. Its main aim is to identify their sources by arrival direction analysis and to measure their energy spectra with a high collecting power, beyond any other previous or planned experiment so far. In addition, five exploratory objectives were defined [4]: (i) the detection of extreme energy gamma rays; (ii) the detection of extreme energy neutrinos; (iii) exploratory studies of the galactic magnetic fields; (iv) fundamental physics 


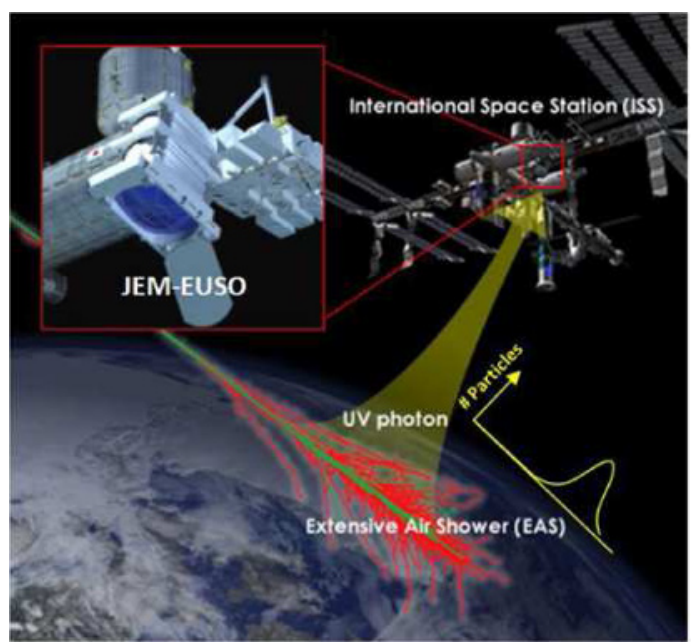

Figure 2. Principle of the JEM-EUSO mission to detect extreme energy cosmic rays via the fluorescence emission of extensive air showers in the Atmosphere.

studies (e.g. Lorentz invariance tests) at extreme energies; (v) global survey of nightglows, plasma discharges, lightning, meteors and other terrestrial transient sources in UV light.

\section{The JEM-EUSO science instrument}

The JEM-EUSO instrument [5] consists of the telescope, the focal surface, a monitoring system for the atmospheric conditions, and a calibration system. In addition, there will be support and calibration systems on ground as well as at the ISS. The main telescope (Fig.3) is a $\mu$ s-fast and highly pixelized digital camera with a diameter of about $2.5 \mathrm{~m}$ and $\mathrm{a} \pm 30^{\circ}$ wide field of view. It works in the near-UV wavelength $(290-430 \mathrm{~nm})$ and enables a single-photon counting. The optics with three Fresnel lenses focuses the incident UV photons onto the focal surface with an angular resolution of $0.07^{\circ}$

The focal surface will consist of 4932 64-channel multianode photomultipliers from Hamamatsu. They will be organized in elementary cells of 4 PMs each and in 137 photo detection modules (PDM) of 9 elementary cells each. A PDM can be seen as an independent unit one of which will also be used for each of the test experiments (see section 4). The focal surface comprises therefore more than 300,000 pixels in total. The electronics process triggers for air-shower or other transient events in the Atmosphere and send necessary data to the ground for further analysis.

The Atmospheric Monitoring System (AMS) consists of an infrared camera and a LIDAR (LIght Detection And Ranging) system, both monitoring the Earth's atmosphere continuously inside the FoV of the JEM-EUSO telescope. A measurement of clouds top heights with an accuracy better than $500 \mathrm{~m}$ will be possible. The calibration system measures continuously the efficiencies of the optics, the focal surface detector, and the data acquisition electronics.

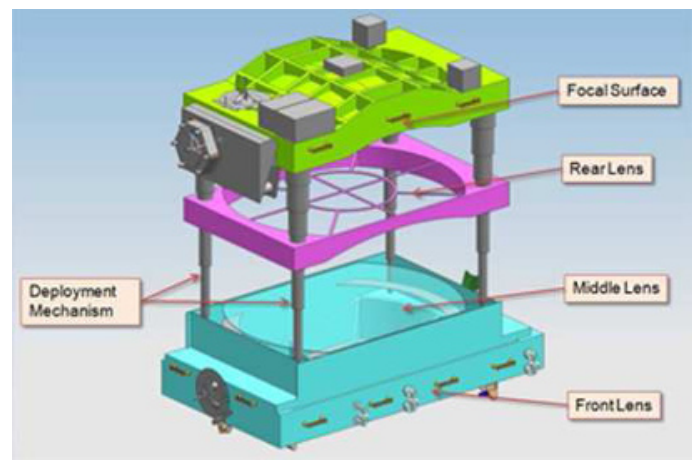

Figure 3. Schematic view of the JEM-EUSO instrument. The dimensions are $2.65 \mathrm{~m}$ times $1.90 \mathrm{~m}$ times approximately $3 \mathrm{~m}$.

\section{Performance of JEM-EUSO}

Fig. 4 shows a typical longitudinal profile of an EAS at the pupil of the telescope with and without clouds. For clear atmospheric conditions a reconstruction of the shower geometry and primary energy with good accuracy, as well as an estimate of the shower maximum will be possible [6]. In presence of optically thin clouds at high altitudes (the case of cloud-height $H_{c}=10 \mathrm{~km}$ and optical thickness of the cloud $\tau_{c}=0.2$ is shown in Fig. 4), often also categorized as cirrus, most of the EAS photons penetrate the layer of clouds and are attenuated only partly. This may lead to a reconstruction with a lower energy, however, the situation can be taken into account as the clouds are monitored by the AMS and the information is known for each triggered EAS. The geometry of the shower axis can still be properly determined by the analysis of the angular velocity of the EAS signal. In case of optically thick clouds,

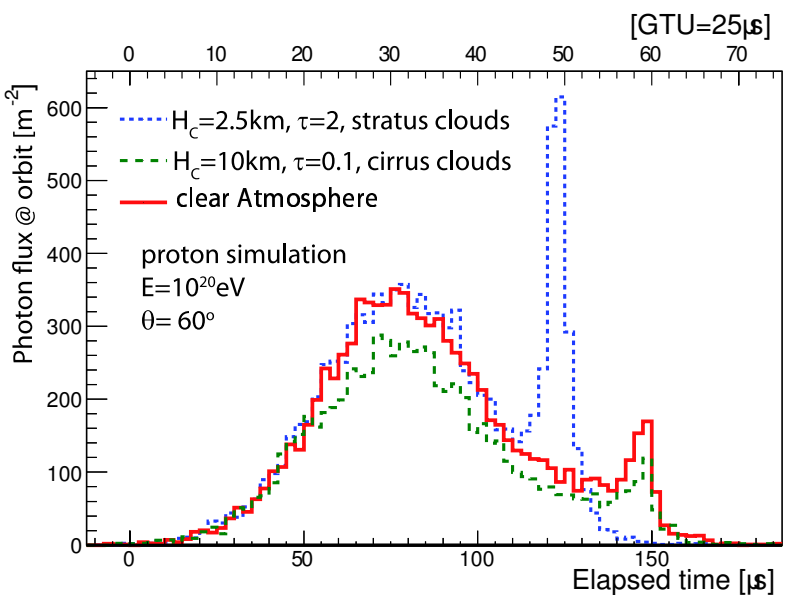

Figure 4. Arrival time distribution of photons at the pupil of the telescope per $\mathrm{m}^{2}$ from an EAS under different cloud conditions. Dashed and dotted lines correspond to the case of cirrus- and stratus-like test clouds, respectively. Solid line is for the case of a clear atmosphere. The Cherenkov peak at the end of the shower profile is located closer to the shower maximum in case of stratus clouds as the light is reflected by the cloud-top instead by the ground level. 


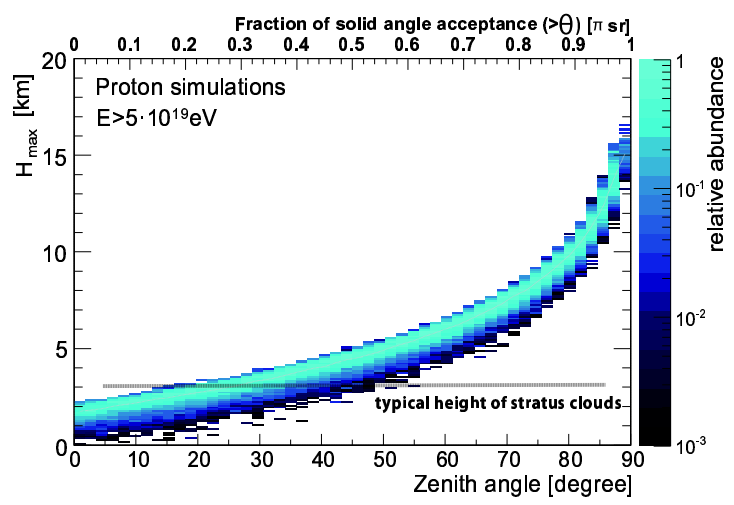

Figure 5. Illustration of the minor effect on the JEM-EUSO reconstruction of optically thick clouds. Shown is the shower maximum distribution of high-energy protons as a function of the incident zenith angle. It can be seen that most EAS with zenith angles larger than $40^{\circ}$ will have the shower maximum above the typical stratus clouds, and therefore the reconstruction will not be significantly affected by the presence of such clouds.

such as stratus clouds, the effect on the photon generation and propagation during the shower development is much larger if the shower maximum lies within the cloud. However, such clouds are mainly at lower altitudes and, as JEM-EUSO has a higher efficiency for inclined showers, in most cases the shower maximum is still above the clouds (Fig. 5). That means that the main part of the shower development is well measured and this allows us to reconstruct the energy deposit in the atmosphere reasonably well. Moreover, the diffusively reflected Cherenkov light enhances the total intensity from the shower, which even increases the trigger efficiency at lower energies. This denotes a substantial difference to ground-based fluorescence observatories: the signals of EAS from higher altitudes are efficiently observed with no or limited attenuation in cloudy cases if either thick clouds are at low altitudes or optically thin clouds are present at high altitudes.

To reach the goals of JEM-EUSO, high statistics and therefore a large annual exposure are required. At the time of writing, detailed simulation studies only for the case of nadir observations (looking vertically down to Earth) with the JEM-EUSO telescope at the ISS were available [7]: The annual exposure depends on zenith angle and primary energy and is determined by four factors:

$$
T A \times \eta \times \kappa \times l
$$

with

\section{- $T A$ : trigger aperture}

The trigger efficiency of the instrument depends strongly on the characteristics of the optics and focal surface (FS) detector, as well as the darkness of the atmosphere. Main objective of the trigger system is the reduction of the rate of fake triggers due to background fluctuations to $<0.1 \mathrm{~Hz}$. Therefore, the trigger logic was defined as a compromise between the available power and telemetry budgets of the instrument, the response of the detector, and the necessity of coping with back- ground fluctuations that could mimic the presence of a signal from an EAS. The rejection power depends also on the average night-glow background, where the background is assumed to be $500 \mathrm{ph} / \mathrm{m}^{2} / \mathrm{ns} / \mathrm{sr}$ (obtained by measurements of the Tatiana satellite in 2012 [8]). Severe high quality conditions are applied to the data. These quality cuts take also into account the better performance of the optics in the center of the FoV and for showers with inclined zenith angles $\left(\theta>60^{\circ}\right.$ for nadir observation mode), which produce longer and less attenuated tracks. The rejection level of the trigger algorithm determines the aperture of the instrument as a function of the energy. A $100 \%$ trigger efficiency can be reached for $E>4 \cdot 10^{19} \mathrm{eV}$ when $\theta>60^{\circ}$ and distance of the shower core to the center of the FoV $R<150 \mathrm{~km}$. For the full field of view there is still $90 \%$ trigger efficiency for cosmic ray events above an primary energy of $E>10^{20} \mathrm{eV}$.

\section{- $\eta$ : observational duty cycle}

An essential parameter to estimate the JEM-EUSO exposure is the fraction of time during which EAS observation is not hampered by the brightness of the atmosphere. Nighttime is defined as the absence of Sun in the visible sky at the orbit level. We take into account the main component of the intensity of diffuse background light, a quantity which is variable over time. The observational duty cycle is defined as the fraction of time during which the background intensity is lower than a given threshold value of the background, where moonlight is the largest component. Moonlight contamination from the phase of the Moon together with its apparent position as seen from the ISS is considered [7, 9, 10]. The fraction of time during which the background is less than 1500 photons per $\mathrm{m}^{2} / \mathrm{ns} / \mathrm{sr}$ is about $20 \%$. This is a conservative estimate as the instrument is not limited by this value, so, for the highest energies measurements can be performed even in a higher background condition.

\section{- $\kappa$ : cloud inefficiency}

In order to quantify the reduction on the effective instantaneous aperture of the telescope due to the presence of clouds, a study on the distribution of clouds as a function of altitude, optical depth and geographical location has been performed using different meteorological data sets $[7,11]$ (TOVS, ISCCP and CACOLO data sets) obtaining similar results on the cloud occurrence. In the calculations the ratio between the aperture when the role of clouds is included compared to pure clear sky, for all events and for those events which have good quality characteristics (optical thickness and height of clouds, or shower maximum well above the cloud top height) are included. More details can be found in [7, 12]. From these results we conclude that $70 \%$ is a conservative estimate of the fraction of the full aperture in which the measurement will not be hampered by atmospheric factors.

\section{- $l$ : city lights and lightnings}

In addition to the diffuse sources of background, there are intermittent local sources such as lightening flashes, 


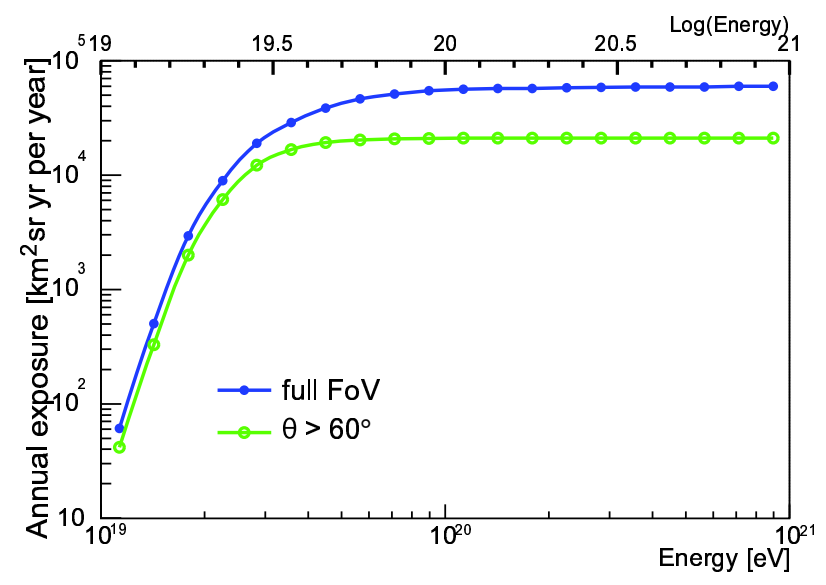

Figure 6. Annual exposure of JEM-EUSO (ISS at $400 \mathrm{~km}$ ) for the full FoV and for the case of extremely high quality cuts applied to the data.

auroras or city lights. To estimate the reduction in observational duty cycle and aperture due to the occurrence of auroras, we use the so-called Kp-index to describe the geomagnetic activity. The data during the years 2001 and 2006 for the respective geomagnetic latitude and longitude of the ISS positions were taken into account. These two years were selected as they were close to solar minimum and solar maximum, respectively. In the estimation, it was assumed that no measurement can be performed when the Kp-index for ISS geomagnetic latitude is equal or higher than Auroral Boundary Index [13]. Even in the case of maximum solar activity the effect is not larger than $1 \%$. Inefficiencies due to the overflight of cities were calculated to a $7 \%$ reduction in observational duty cycle and instantaneous aperture. In the cases of lightning strokes and other transient luminous events (TLE), estimates are performed assuming the rate of events detected by Tatiana satellite [8]. We further assume that each event seen by JEMEUSO prevents cosmic ray measurement in the entire FoV for $70 \mathrm{~s}$, which corresponds to the time needed to completely change the FoV. Even with these extremely conservative assumptions, the overall effect is less than $2 \%$. Moreover, as lightning strikes are very often associated with high altitude clouds, most of these are already included in the estimate of the cloud inefficiency.

As result, Fig. 6 shows that $80-90 \%$ of the full exposure is already reached at energies $E=3 \cdot 10^{19} \mathrm{eV}$ when we rely on incident zenith angles larger than $60^{\circ}$. Using all events, JEM-EUSO has an annual exposure of $60 \cdot 10^{3} \mathrm{~km}^{2}$ sr year at $10^{20} \mathrm{eV}$. JEM-EUSO will overlap in the accessible energy range with ground based experiments to crosscheck systematics and performances. At higher energies JEM-EUSO will be able to accumulate statistics yearly of about one order of magnitude higher than currently existing ground based detectors. Later, JEM-EUSO is foreseen to be operated also in tilt mode to further increase the exposure at the highest energies $\left(>10^{20} \mathrm{eV}\right)$ by a factor of at

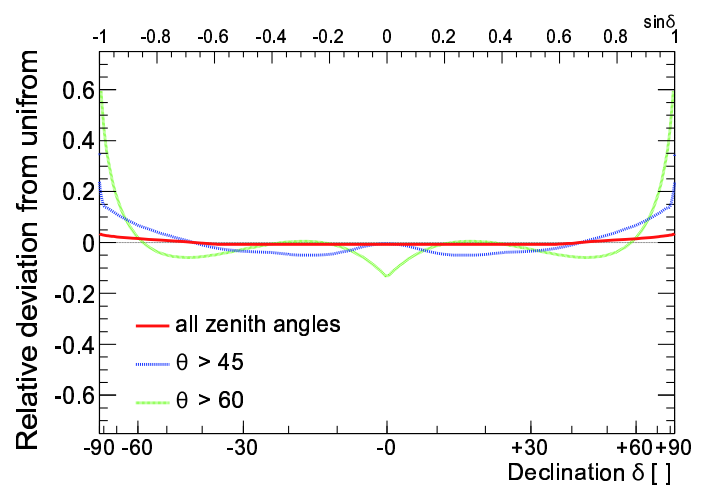

Figure 7. Relative deviation from uniformity of the aperture as a function of sine of declination. Dashed curves show the cases for a selection of events with different zenith angle. The pure isotropic exposure to solid angle is defined as 0 . The horizontal axis on the bottom denotes corresponding declination.

least three compared to nadir mode. The optimization of the tilt parameters is still under evaluation.

Finally, Fig. 7 demonstrates the uniformity of exposure expected for the JEM-EUSO mission as a function of sine of declination (solid angle). The orbiting JEM-EUSO telescope will cover the entire celestial sphere. This will guarantee observing both hemispheres with similar systematic uncertainties, avoiding possible inconsistencies in the results of instruments looking at different portions of the sky.

\section{Test Experiments}

Two test or pathfinder experiments are currently under development, a ground-based telescope (TA-EUSO) and a space-based one (EUSO-Balloon), both equipped with one full original JEM-EUSO PDM (photon detection module with 2304 pixels) and with adapted new optical systems for the respective purpose.

EUSO-Balloon [14] will serve as a demonstrator for technologies and methods featured in the space instrument (Fig. 8). This balloon-borne instrument points towards the nadir from a float altitude of about $40 \mathrm{~km}$. With its Fresnel optics and PDM, the instrument monitors a 12 by 12 degree wide field of view. The instrument is presently built.
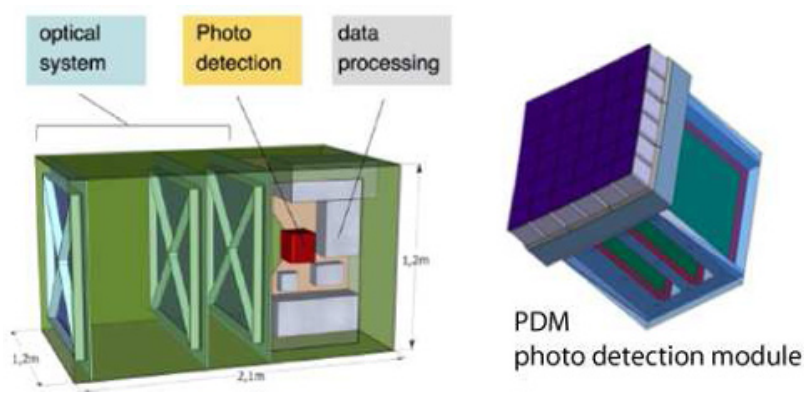

Figure 8. Schematic view of the EUSO-Balloon instrument and the Photo Detection Module (PDM) used as focal surface instrumentation (also for TA-EUSO). 


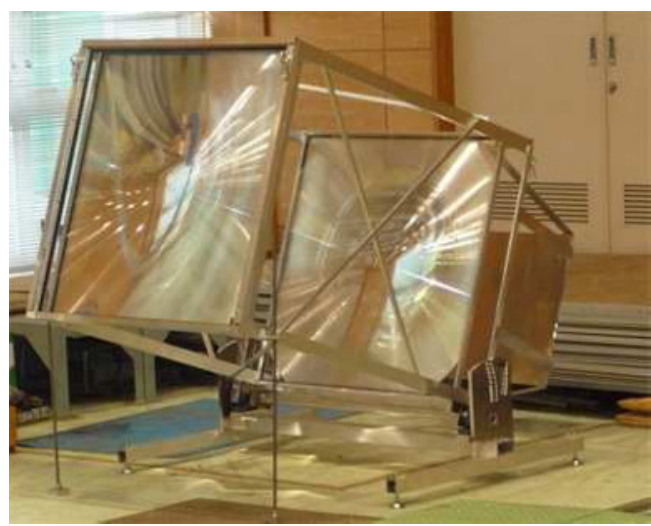

Figure 9. Photograph of the lenses system which will be used for TA-EUSO.

The flights will be performed by the balloon division of the French Space Agency CNES. A first flight is scheduled in 2014. Key objectives are among others the full-scale endto-end test of the JEM-EUSO technique, the experimental determination of the effective UV background below 40 $\mathrm{km}$, ground based calibration systems, and, the observation of the first UV-image of an EAS looking down on the Earth's atmosphere.

Even before the first balloon launch, important information on the capabilities of the instrument will be provided by TA-EUSO [15], which is a ground-based telescope formed by one PDM (identical to the PDM of EUSO-Balloon) and two Fresnel lenses, which are prototypes of those foreseen in JEM-EUSO (Fig. 9). TA-EUSO will be located at the Telescope Array (TA) [16] site in Utah, USA and will be installed in early 2013. The instrument will measure the UV light in the Atmosphere in its \pm 4 degrees FoV, will be triggered by the fluorescence detectors of TA, and will make use of the LIDAR and the Electron Light Source of TA. The aims are to obtain an end-to-end calibration of the prototype telescope, and an inter-calibration with the fluorescence detector of TA.

\section{Conclusion}

The JEM-EUSO mission is planned as a three to five year mission following deployment on the ISS in 2017. JEMEUSO triggers on the UV light from EAS, measures the intrinsic luminosity of each EAS near its maximum and accurately reconstructs the EAS path so that the arrival direction of the initiating EECR can be reconstructed. Current studies [17] indicate that individual showers can be reconstructed with a $68 \%$ separation angle less than $2.5^{\circ}$. Regarding the energy reconstruction, at the current status of development of the reconstruction algorithms, proton showers are reconstructed in clear-sky conditions with a typical energy resolution $\Delta E / E$ of $\approx 25 \%(20 \%)$ at energies around $4 \cdot 10^{19} \mathrm{eV}\left(10^{20} \mathrm{eV}\right)$. In addition, our still preliminary results indicate that the $X_{\max }$ resolution can be better than $70 \mathrm{~g} / \mathrm{cm}^{2}$ for $E>10^{20} \mathrm{eV}$. With $0.1-1$ million $\mathrm{km}^{2} \mathrm{sr}$ exposure and the uniform coverage of the full sky, JEMEUSO will observe all possible source directions at least within several hundred Mpc and will make possible the (i) identification of sources with unprecedentedly high statistics by arrival direction analysis and (ii) the measurement of the energy spectra from individual sources to constrain the acceleration or the emission mechanisms.

The test experiments presently under construction will provide more information and details on the capabilities of the JEM-EUSO mission in near future.

The corresponding author acknowledges support from the 'Helmholtz Alliance for Astroparticle Physics HAP' funded by the Initiative and Networking Fund of the Helmholtz Association, Germany.

\section{References}

[1] T. Ebisuzaki et al., Nucl. Phys. B (Proc. Suppl.) 175 (2008) 237.

[2] Y. Takahashi et al., New J. Phys. 11 (2009) 065009.

[3] M. Casolino et al., Astrophys. Space Sci. Trans 7 (2011) 477.

[4] G. Medina-Tanco et al., Proc. 32nd Int. Cosmic Ray Conf., Beijing (2011) Vol.3, p.204; (preprint) arXiv:1204.5065.

[5] F. Kajino et al., Nucl. Inst. Meth. A 623 (2010) 422.

[6] F. Fenu et al., Proc. 32nd Int. Cosmic Ray Conf., Beijing (2011) Vol.3, p.116; (preprint) arXiv:1204.5065.

[7] J. Adams et al. - JEM-EUSO Collaboration, Astropart. Phys. (2013), submitted 2012.

[8] G.K. Garipov et al., Astropart. Phys. 24 (2005) 400.

[9] http://sscweb.gsfc.nasa.gov/cgi-bin/Locator.cgi

[10] F. Montanet, EUSO-SIM-REP-009-1.2 2004.

[11] F. Garino et al., Proc. 32nd Int. Cosmic Ray Conf. , Beijing (2011) Vol.3, p.88; (preprint) arXiv: 1204.5065.

[12] G. Saez Cano et al., Proc. 32nd Int. Cosmic Ray Conf., Beijing (2011) Vol.3, p.231; (preprint) arXiv:1204.5065.

[13] http://www.swpc.noaa.gov/Aurora/index.html

[14] P. Von Ballmoos et al., Proc. UHECR2012, CERN$\mathrm{CH}$, in press

[15] M. Casolino et al., Proc. UHECR2012, CERN-CH, in press.

[16] T. Abu-Zayyad et al., Astropart. Phys. 39 (2013) 109.

[17] M. Bertaina et al., Contribution to a special issue of 'Advances in Space Research' on Cosmic Ray Origins: Viktor Hess Centennial Anniversary (2013), submitted. 